\title{
ANALYSIS OF SOIL FREEZE/THAW SIGNATURES DURING SLAPEX F/T CAMPAIGN
}

\author{
Edward Kim* (l), Tracy L. Rowlandson ${ }^{2}$, Aaron A. Berg ${ }^{2}$, Alexandre Roy ${ }^{3}$, Renato Pardo ${ }^{2}$, Jarrett \\ Powers $^{4}$, Paul Houser ${ }^{5}$, Kyle McDonald ${ }^{6}$, Peter Toose ${ }^{7}$, Albert Wu $u^{1,8}$, Eugenia De Marco ${ }^{1,8}$ Chris \\ Derksen ${ }^{7}$, Yiwen Zhou ${ }^{9}$, Roger Lang ${ }^{9}$, Jared Entin ${ }^{10}$, Kristin Lewis ${ }^{10}$
}

(1) NASA Goddard Space Flight Center; (2) University of Guelph, (3) Université de Sherbrooke, (4) Agriculture and Agri-Food Canada, (5) George Mason University, (6) City College of New York, (7) Environment and Climate Change Canada, (8) ATA Aerospace, (9) George Washington University, (10) NASA Headquarters

\begin{abstract}
Permanently frozen and seasonally frozen soils occur over a large portion of the Earth's land surface. Changes in the freeze/thaw state of the land surface reflects major changes in thermal and hydraulic properties as well as acting as a "switch" for many ecological processes. In short, soil freeze/thaw state is a fundamental land surface variable in the water and energy cycles, and it connects to the carbon cycle.

Surface freeze/thaw state is observable by passive and active microwave sensors. For example, NASA's Soil Moisture Active Passive (SMAP) mission includes a freeze/thaw data product. Such satellite sensing offers routine all-season and all-weather global observations of soil freeze/thaw state with the application of suitable algorithms.

We describe early finding from the SLAPex Freeze/Thaw campaign, believed to be the first airborne campaign of its type, focusing on soil freeze/thaw.
\end{abstract}

Index Terms - frozen soil, soil moisture, freeze/thaw, permafrost, SLAP

\section{INTRODUCTION}

Up to a third of Earth's land surface experiences either seasonal freezing of just the surface soil or the converse-permafrost, with seasonal thawing of just the surface active layer [1].

Thermal and hydraulic properties of soils can change by orders of magnitude during freeze/thaw transitions [2]. Such significant changes, in turn, can exert a huge control on the annual thermal and hydraulic regimes of such areas [3]. Infiltration, runoff, plant growth, depth of soil freeze, and depth of active layer thaw are just some of the affected processes.

The importance of knowing the surface freeze/thaw state is recognized, for example, in the goals of NASA's Soil
Moisture Active Passive (SMAP) mission, which includes a soil freeze/thaw data product [4] that is now derived from the SMAP L-band radiometer after the demise of the SMAP radar. The larger footprint size of the SMAP radiometer $(\sim 36 \mathrm{~km}$ diameter) introduces additional scaling questions when applying the freeze/thaw algorithm. For example, how much of the footprint needs to freeze for the algorithm to detect a frozen state? And, even for a footprint whose entire area is frozen, how deep does the frozen soil need to be for the algorithm to detect it? Answering these questions requires both wide area observations to address scaling issues and point-scale time series observations to understand the correspondence between microwave signatures and the soil freeze/thaw at the process-level. However, the SMAP validation plans include no field campaigns for freeze/thaw validation [5].

\section{GOAL OF SLAPEX FREEZE/THAW}

Investigating the L-band microwave signatures of soil freezing and thawing, both over an area as well as with time series was the original scientific motivation for the SLAPex Freeze/Thaw campaign. The joint US-Canada effort included three L-band sensors: an airborne sensor to address the spatial and temporal questions over large areas, plus two ground-based L-band radiometers to provide temporal observations for process-level understanding. This was unique since we know of no prior field campaigns focused on soil freeze/thaw that have combined airborne wide area sensing as well as point scale time series observations (a Chinese campaign addressed the entire annual hydrologic cycle [6]).

While the motivation behind SLAPex Freeze/Thaw was not driven by SMAP's needs, it is obvious that one use of such field observations is for validation type studies of freeze/thaw algorithms, such as the one used by SMAP. 


\section{STUDY AREA DESCRIPTION}

SLAPex Freeze/Thaw took place over agricultural and pasture fields near Winnipeg, Manitoba, Canada during the first half of November, 2015. The area is coincident with the area used by the SMAPVEX12 and SMAPVEX16 soil moisture campaigns. Agriculture and Agri-Food Canada also operates a network of permanent soil moisture stations in the area that are a core validation site for the SMAP mission.

The area is flat, and the soils range from coarse to silty clay loam to heavy clay. Soil texture had been previously characterized. 18 fields were observed by the airborne sensor and ground truth teams. 15 of these sites had been harvested and were essentially bare soil. The remaining 3 sites were pasture.

\section{GROUND TRUTH OBSERVATIONS}

Detailed ground truth was collected by a team of researchers from US and Canadian institutions. These truth data included soil temperature at various depths, freeze/thaw state, soil moisture, characterization of surface roughness, vegetative matter (e.g., crop litter). The data collected by field teams was supplemented by automated in situ sensors. Ground truth observations were made in the early morning before sunrise and in the afternoon, to coincide with frozen and thawed conditions, respectively.

\section{GROUND-BASED SENSING}

Point-scale continuous time series observations were provided by two tower-based L-band radiometers-one from Environment and Climate Change Canada and the other from the University of Sherbrooke. These continuous observations provide temporal context between airborne observations as well as providing the smallest-footprint microwave observations of freezing and thawing events.

These time series data will be described for what they show by themselves as well as in relation to the airborne and satellite L-band observations at very different spatial scales.

\section{AIRBORNE SENSING}

The airborne microwave observations were provided by NASA Goddard's Scanning L-band Active Passive (SLAP) instrument. With both a radiometer and a scatterometer, the same polarizations, incidence angle, and conical scan geometry as SMAP, SLAP is an airborne simulator of the spaceborne SMAP.

Thirteen science flights were flown over the study area. Five lines were flown each flight to correspond with ground truth sample locations and each day two flights were conducted in frozen (AM) and thawed (PM) conditions, coinciding with ground truth collection times. Two additional "mow-the-lawn" (MTL) flights were flown to obtain full coverage of a $36 \times 36 \mathrm{~km}$ SMAP pixel, the first under thawed (morning) and the second under frozen (afternoon) conditions.

Details of SLAP, its performance and the calibration of the radiometer and radar will all be presented.

\section{COMPARISON WITH SATELLITE OBSERVATIONS}

Figures $1-4$ show calibrated, coincident SLAP radiometer and radar imagery covering one 36 x $36 \mathrm{~km}$ SMAP pixel from an MTL flight on November 8, 2015 when thawed conditions prevailed. Footprints were 400x800 m ovals.

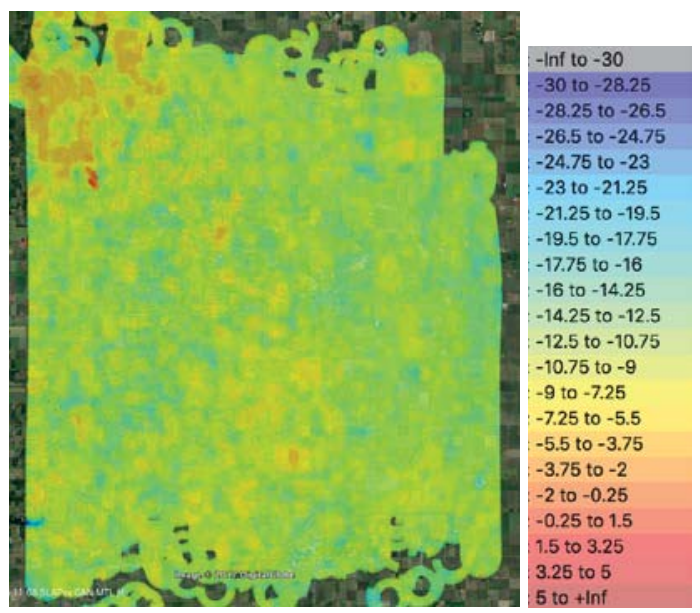

Figure 1. H-pol SLAP radiometer TB image of 36x $36 \mathrm{~km}$ SMAP pixel.

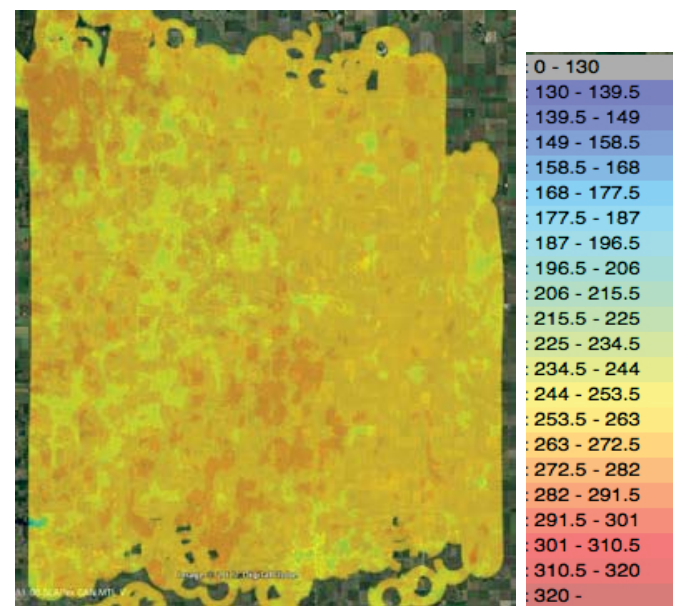

Figure 2. V-pol SLAP radiometer TB image of 36x $36 \mathrm{~km}$ SMAP pixel. 


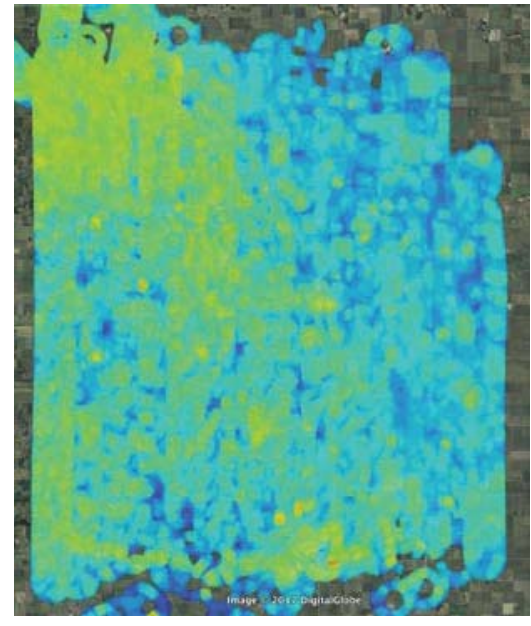

Figure 3. HH-pol SLAP scatterometer sigma0 image of 36x 36 km SMAP pixel.

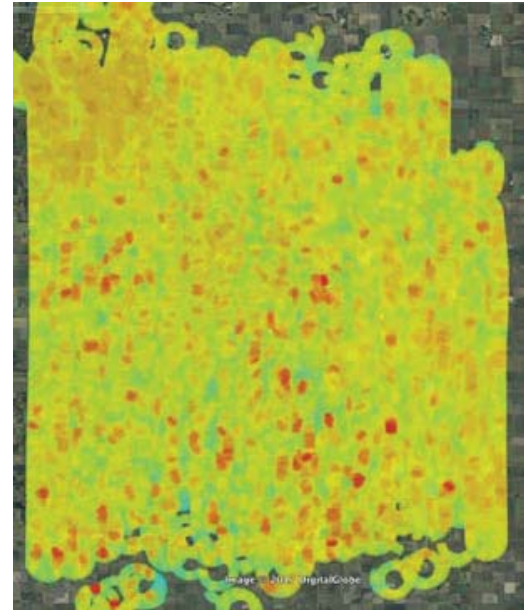

- Inf to -30
-30 to -28.25
-28.25 to -26.5
-26.5 to -24.75
-24.75 to -23
-23 to -21.25
-21.25 to -19.5
-19.5 to -17.75
-17.75 to -16
-16 to -14.25
-14.25 to -12.5
-12.5 to -10.75
-10.75 to -9
-9 to -7.25
-7.25 to -5.5
-5.5 to -3.75
-3.75 to -2
-2 to -0.25
-0.25 to 1.5
1.5 to 3.25
3.25 to 5
55 to + Inf

Figure 4. VV-pol SLAP scatterometer sigma0 image of 36x 36 km SMAP pixel.

\section{CONCLUSIONS}

The SLAPex Freeze/Thaw campaign employed airborne and tower-based L-band sensors to investigate the microwave signatures of soil freeze/thaw transitions, and to relate these to actual in situ conditions known from ground truth observations. This unique dataset is being used to answer basic questions about the sensitivity of remote sensing to freeze/thaw extent and depth. These results, in turn, can inform the refinement of freeze/thaw algorithms used by low-frequency satellites to provide regular global data products of soil freeze/thaw state.

Example airborne and tower microwave data will be presented and discussed. SLAP details and calibration will be presented, and implications for SMAP-like freeze/thaw algorithms will be discussed.

\section{REFERENCES}

[1] Williams, P. J., and M. W. Smith, The Frozen Earth: Fundamentals of Geocryology, Cambridge University Press, Cambridge, 1989.

[2] O. T. Farouki, "Thermal properties of soils," CRREL Monograph 81-1, U. S. Army Cold Regions Research and Engineering Lab., Hanover, NH, 1981.

[3] L. Hinzman, D. Kane, R. Gieck, and K. Everett, "Hydrologic and thermal properties of the active layer in the Alaskan arctic," Cold Regions Sci. and Tech., vol. 19, pp. 95-110, 1991.

[4] C. Derksen, et al, "Retrieving landscape freeze/thaw state from Soil Moisture Active Passive (SMAP) radar and radiometer measurements," Remote Sens. Environ. 194, pp. 48-62, 2017.

[5] A. Colliander, et al,. "Validation of SMAP surface soil moisture products with core validation sites," Remote Sens. Environ. 191, pp. 215-231, 2017.

[6] X. Li, et al, "Watershed Allied Telemetry Experimental Research,” JGR, Vol 114, D22103, 2009. 